\title{
The roller-coaster ride of thyroid function before, during and after pregnancy
} S Wynne, S Mwenechanya, M Banerjee

\section{Diabetes \& Endocrine Unit \& Division of Women's health, Arrowe Park Hospital}

Introduction: Hyperthyroidism is a common endocrine disorder in pregnancy, with potentially significant foeto-maternal consequences.

Description of the case: 25 year old lady with auto-immune hyperthyroidism (TRAb>400) was treated with Propranolol and Carbimazole and referred to the Endocrine clinic. She was started on Block and replace therapy. She was soon moved over to Propylthiouracil as she was contemplating pregnancy. She became hypothyroid within 3 months and anti-thyroid therapy was stopped. In another 2 months she required thyroxine replacement. 3 months later, her TSH started to drop and by 12 months, she was found to be hyperthyroid and Carbimazole was started. 2 months later, she was found to be pregnant and was again changed to PTU. During the rest of her pregnancy, her FT3 remained high, FT4 - normal with TSH undetectable, despite treatment with PTU $300 \mathrm{mg}$ bd with regular counselling. She delivered a healthy but hyperthyroid baby (FT4: 64, FT3: 31.3, TSH<0.001, TRAb > 400 ) of birthweight 3150 grams at 37 weeks +1 day of gestation. Mum's TFTs 1 day postnatal were still in the hyperthyroid state, but when checked after 5 weeks, showed features of hypothyroidism with TSH 9.11. Her PTU was then reduced to $100 \mathrm{mg} /$ day, but she became grossly hypothyroid in 2 months, needing Thyroxine replacement. Within further 2 months, her hyperthyroidism flared up again and is currently being managed by block \& replace regimen. The infant is now being treated with Carbimazole and is euthyroid.

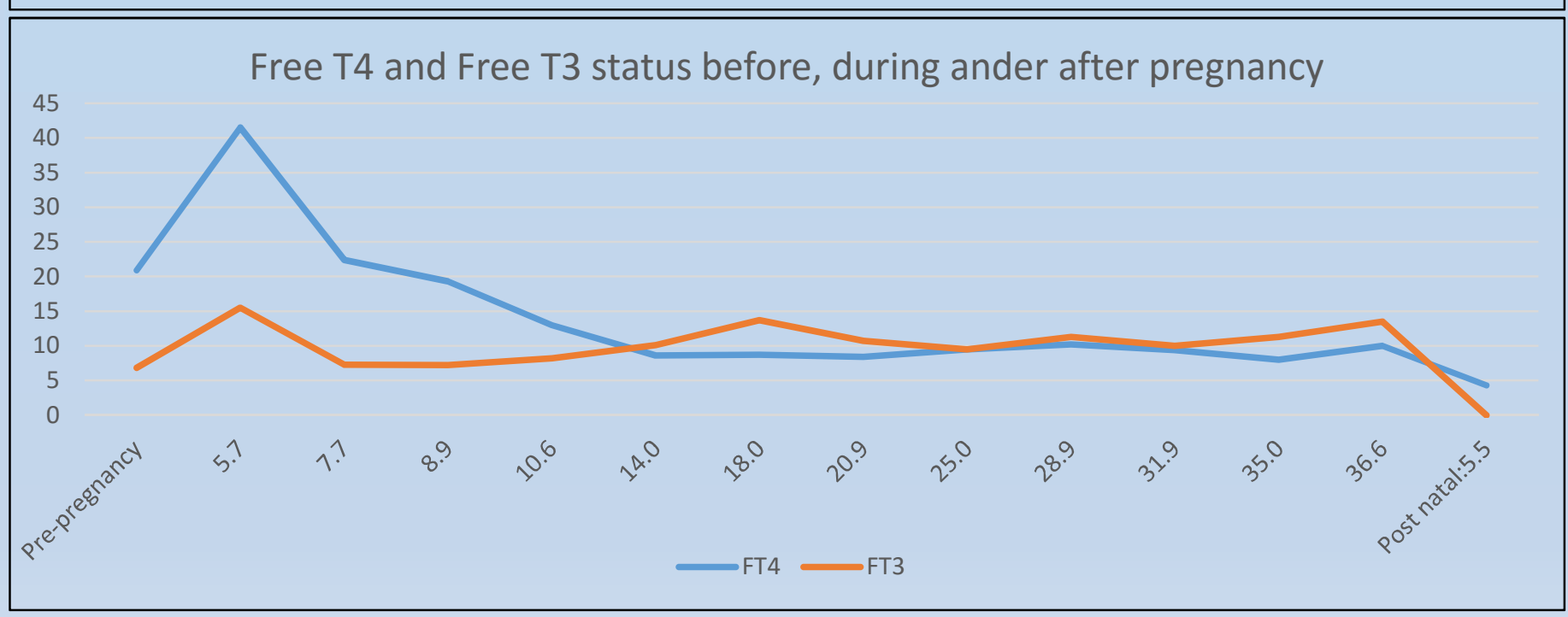

\section{Discussion:}

This case brings forward the close monitoring required in such patients during and after pregnancy. It also highlights the following:

The role of anti-TSH receptor antibodies in fluctuation of thyroid status:

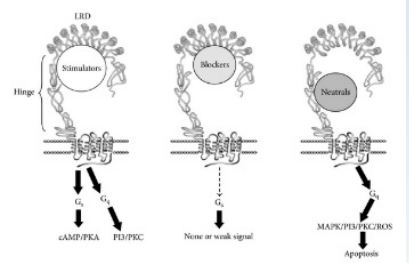

The binding sites of the stimulating and blocking antibodies on the TSH-receptors are completely different. The factors affecting this differential binding is not known.
The contribution of placental glucuronidase system in degradation of PTU: Apart from liver, the UDPglucuronidase system, which metabolises PTU is found mainly in the placenta.

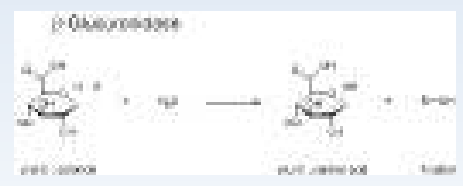

The relative lack of efficacy of PTU seen in this case during pregnancy, which returned after delivery, could be due to accelerated catabolism of PTU by placental glucuronidase system. 